\title{
DNAJC3 mutation in Thai familial type 2 diabetes mellitus
}

\author{
SIRIKUL KULANUWAT ${ }^{1,2}$, WATIP TANGJITTIPOKIN ${ }^{1,2}$, PRAPAPORN JUNGTRAKOON ${ }^{1,3}$ \\ CHUTIMA CHANPRASERT ${ }^{1,2,4,5}$, JATUPORN SUJJITJOON ${ }^{1,3}$, NINAREEMAN BINNIMA ${ }^{1,2}$, \\ PA-THAI YENCHITSOMANUS ${ }^{1,3}$ and NATTACHET PLENGVIDHYA ${ }^{1,5}$ \\ ${ }^{1}$ Siriraj Center of Research Excellence for Diabetes and Obesity, ${ }^{2}$ Department of Immunology, \\ ${ }^{3}$ Division of Molecular Medicine, Department of Research and Development, ${ }^{4}$ Research Division, \\ and ${ }^{5}$ Division of Endocrinology and Metabolism, Department of Medicine, \\ Faculty of Medicine Siriraj Hospital, Mahidol University, Bangkok 10700, Thailand
}

Received December 1, 2017; Accepted May 8, 2018

DOI: $10.3892 /$ ijmm.2018.3678

\begin{abstract}
Type 2 diabetes mellitus (T2D) is a heterogeneous disease, with certain cases presenting an autosomal dominant type. The rare coding variants of disease-causing genes in T2D remain mostly unclear. The present study aimed to identify the disease-causing gene conducting whole exome sequencing in a Thai T2D family with an autosomal dominant transmission of T2D with no evidence of mutations in known maturity-onset diabetes of the young (MODY) genes. Candidate variants were selected according to certain criteria of mutation prediction programs, followed by segregation analysis with diabetes in the family. The results demonstrated that, of the 68,817 variants obtained, 122 were considered as candidate variants subsequent to the filtering processes. Genotyping of these variants revealed that DnaJ homolog subfamily $\mathrm{C}$ member 3 (DNAJC3) p.H238N segregated with diabetes in the family. This mutation was also identified in another proband from the autosomal dominant T2D family without mutation in known MODY genes and was segregated with diabetes. This variant was also identified in 14/1,000 older-onset T2D patients [minor allele frequency $(\mathrm{MAF})=0.007], 2 / 500$ non-diabetic controls $(\mathrm{MAF}=0.002)$ and 3 prediabetic individuals who were previously classified as non-diabetic controls. In silico mutagenesis and protein modeling of p.H238N revealed changes of the polar contacts across the tetratricopeptide repeat (TPR) motif and TPR subdomains, which may affect the protein tertiary
\end{abstract}

Correspondence to: $\operatorname{Dr}$ Nattachet Plengvidhya, Division of Endocrinology and Metabolism, Department of Medicine, Faculty of Medicine Siriraj Hospital, Mahidol University, 2 Wanglang Road, Bangkoknoi, Bangkok 10700, Thailand

E-mail: sinpv.natpl@gmail.com

Dr Watip Tangjittipokin, Department of Immunology, Faculty of Medicine Siriraj Hospital, Mahidol University, 2 Wanglang Road, Bangkoknoi, Bangkok 10700, Thailand

E-mail: watip.tan@mahidol.edu

Key words: familial type 2 diabetes mellitus, autosomal dominant inheritance, DnaJ homolog subfamily $\mathrm{C}$ member 3 , exome sequencing structure. Furthermore, the expression of DNAJC3 H238N protein was $0.68 \pm 0.08$ fold $(\mathrm{P}<0.05)$ lower when compared with that of the wild-type, possibly due to protein instability. Thus, $D N A J C 3$ p.H238N is likely to be a variant causing diabetes.

\section{Introduction}

Diabetes mellitus (DM) is a common metabolic disorder characterized by chronic hyperglycemia. The prevalence of DM is increasing worldwide, and the International Diabetes Federation has estimated that 592 million people will suffer from diabetes by 2035 (1). DM-associated chronic complications cause considerable morbidity and mortality (2), and the treatment of these complications imposes an enormous economic burden on healthcare systems. The most common form of DM is type 2 DM (T2D), accounting for $90 \%$ of cases. It has been demonstrated that genes serve an important role in the disease development by interacting with environmental factors, such as a sedentary life-style, diet and obesity (3). Although T2D typically begins in middle-aged or older individuals, early-onset T2D at $<45$ years of age has been reported in several ethnic groups (4). Obesity in young adults may be one of the factors driving the earlier onset of T2D (5). The inheritance of a stronger genetic influence within a family is another determinant, with early-onset T2D individuals likely to have a multigenerational family history of diabetes. The most definite form of familial, autosomal dominant inheritance of diabetes is maturity-onset diabetes of the young (MODY). Typically, MODY is characterized by a young age at onset (usually prior to 25 years of age) and an autosomal mode of inheritance in multigenerational pedigrees (6).

The identification of T2D-causing genes will enable precise disease classification, leading to better treatment and prevention of the disease and its chronic complications. In 2007, there was a breakthrough in the identification of T2D genetic risk loci through a genome-wide association study (GWAS), and >100 such loci have been identified to date (7). However, several limitations of GWAS have been proposed; for instance, the associated single nucleotide polymorphisms fall outside coding regions. Numerous low-frequency variants have never been directly tested for an association with the trait 
and they may explain only $\sim 11 \%$ of T2D heritability $(8,9)$. To address this issue, current research is focusing on finding low-frequency to rare variants with intermediate to large effects that are associated with diabetes (10). Recent advances in nucleic acid sequencing by next-generation sequencing technology have facilitated the identification of genes causing diseases (10). Furthermore, whole-exome sequencing (WES) has led to the successful discovery of mutations between rare coding variants and T2D $(11,12)$.

Thus, the aim of the present study was to identify rare variant causing diabetes in Thai T2D families. Through the application of WES, the study identified the causative variant in a large family with autosomal dominant T2D in which a proband developed T2D at an early age.

\section{Materials and methods}

Diabetes families. Diabetes families were recruited at the Diabetic Clinic, Siriraj Hospital, Mahidol University (Bangkok, Thailand) using the following criteria: i) The proband and at least one first-degree relative were diagnosed with diabetes before the age of 35 years; ii) two or more generations were affected by diabetes; iii) glycemic control could be accomplished with diet and/or oral agents; iv) there was no history of diabetic ketoacidosis; and v) the proband was negative for anti-glutamic acid decarboxylase antibody. A total of 91 autosomal dominant diabetes families were recruited between January 2009 and 2017, 42 of whom fitted into the classic MODY criteria, characterized by early age of onset (usually before 25 years) and autosomal mode of inheritance in multigenerational pedigree (13). The selected family was a large family composed of 17 members (Fig. 1A; Table I), in which the proband and third generation family members developed diabetes at a young age of $<35$ years, and diabetes had been transmitted through three generations. This selected family did not fit classic MODY criteria and was one of the 49 early-onset T2D families. The proband and her first degree relative were diagnosed with diabetes before 35 years of age and the diabetes was transmitted in an autosomal dominant fashion. Mutations of 14 known MODY genes [HNF4A (MIM: 600281), GCK (MIM: 138079), HNF1A (MIM: 142410), PDX1(MIM: 600733), HNFIB (MIM: 189907), NEURODI (MIM: 601724), KLF11 (MIM: 603301), CEL (MIM: 114840), PAX4 (MIM: 167413), INS (MIM: 176730), BLK (MIM: 191305), ABCC8 (MIM: 600509), KCNJ11 (MIM: 600937) and APPL1 (MIM: 604299)] were excluded as a cause of diabetes in the family.

Unrelated T2D patients. A total of 1,000 unrelated T2D patients, diagnosed according to the American Diabetes Association (ADA) 2017 criteria (14), were enrolled at the Diabetic Clinic of Siriraj Hospital, Mahidol University. The age at onset was $>45$ years (mean age \pm standard deviation $=54.6 \pm 11.0$ years).

Non-diabetic subjects. In total, 500 non-diabetic subjects with an age of $>40$ years (mean age \pm standard deviation $=50.69 \pm 8.5$ ), were recruited from the health-checkup facility at the Department of Preventive and Social Medicine, Siriraj Hospital, Mahidol University. All participants had a fasting plasma glucose of $<5.55 \mathrm{mmol} / \mathrm{l}(100 \mathrm{mg} / \mathrm{dl})$, a glycated hemoglobin (HbA1c) level of $\leq 38 \mathrm{mmol} / \mathrm{mol}$ (5.6\%), and normal blood pressure. These subjects had no family history of diabetes among their first-degree relatives.

Ethical approval and consent to participate. All subjects were informed of the purpose of the study prior to signing an informed consent form. The entire study was approved by the Ethics Committee, Faculty of Medicine, Siriraj Hospital, Mahidol University (COA no. Si491/2014), and was conducted according to the Declaration of Helsinki, the Belmont Report, Council for International Organizations of Medical Sciences Guidelines and the International Conference on Harmonization in Good Clinical Practice.

Exome sequencing. DNA samples of 2 diabetic and 2 non-diabetic family members were subjected to exome sequencing. Exome capture was performed using the Agilent SureSelect Human All Exon 50 Mb kit (Agilent Technologies, Inc., Santa Clara, CA, USA) according to the manufacturer's protocol. The captured library was then loaded onto the Illumina HiSeq 2000 platform (Illumina, Inc., San Diego, CA, USA) for amplifying and sequencing. The sequence reads were mapped to the reference human genome (UCSC NCBI37/hg19) using the Burrows-Wheeler Aligner (15). Variant detections and annotations were performed by SAMtools (16) and the Genome Analysis Toolkit (17), respectively. Variants with low quality scores and those covered by $<5$ reads were excluded.

Candidate variant selection. Candidate variants were selected using an autosomal dominant mode of inheritance according to the following criteria: i) Variants identified only in diabetic subjects;ii) heterozygous,non-synonymous variants with aminor allele frequency (MAF) of $<0.01$ were selected from the 1000 Genome Project (http://www.1000genomes.org/), the National Institute of Environmental Health Sciences Exome Project (http://evs.gs.washington.edu/niehsExome/) and the dbSNP147 database from UCSC Genome Browser Gateway (http://genome. ucsc.edu/cgi-bin/hgGateway?hgsid=662106975_rQ8ylYRLVtbw0NVxwJnh1 AhFhb5S\& redirect=manual \&source=genome. ucsc.edu); and iii) variants predicted by at least one out of four in silico programs (including MutationTaster, VarioWatch, PolyPhen2 and SIFT) (18) as possible pathogenic mutations, and residing in genes involved in diabetes and metabolism as determined using the GeneDistiller 2014 (http://www. genedistiller.org/) (19). Candidate variants passing the criteria were validated by Sanger sequencing (Macrogen, Inc., Seoul, Republic of Korea). Furthermore, protein stability of candidate variants was predicted by DUET algorithms (http://structure.bioc.cam.ac.uk/duet) (20).

Segregation analysis. The validated candidate variants were investigated for segregation with diabetes in the family by means of polymerase chain reaction (PCR)-restriction fragment length polymorphism. Restriction enzymes for the genotyping of each candidate variant were determined using NEBcutter (http://nc2.neb.com/NEBcutter2/). Candidate variants segregated with diabetes were genotyped in other 90 early-onset T2D/MODY-X patients, 1,000 T2D patients and in 500 non-diabetic subjects. 
Table I. Clinical characteristics and genotypes of each member of the type 2 diabetes families.

A, Family A

\begin{tabular}{|c|c|c|c|c|c|c|c|c|}
\hline Member & Genotype & Sex & $\begin{array}{c}\text { Age at } \\
\text { diagnosis } \\
\text { (years) }\end{array}$ & $\begin{array}{c}\text { BMI } \\
\left(\mathrm{km} / \mathrm{m}^{2}\right)\end{array}$ & $\begin{array}{l}\text { Glycemic } \\
\text { status }\end{array}$ & $\begin{array}{c}\text { FPG } \\
(\mathrm{mmol} / \mathrm{l})\end{array}$ & $\begin{array}{c}\text { HbAlc } \\
(\mathrm{mmol} / \mathrm{mol})\end{array}$ & $\begin{array}{l}\text { Current } \\
\text { treatment }\end{array}$ \\
\hline I1 & $\mathrm{CA}$ & M & 49 & NA & DM & NA & NA & OHA \\
\hline $\mathrm{I} 2$ & $\mathrm{CC}$ & $\mathrm{F}$ & - & 15.3 & NG & 4.848 & 37 & - \\
\hline II1 & $\mathrm{CC}$ & M & 58 & 29.4 & DM & 7.052 & 56 & OHA \\
\hline II2 & $\mathrm{CA}$ & M & 65 & 21.3 & DM & 12.507 & 98 & - \\
\hline II3 & $\mathrm{CC}$ & $\mathrm{F}$ & - & 20.8 & NG & 4.628 & NA & - \\
\hline II4 & $\mathrm{CA}$ & M & 62 & 24.2 & $\mathrm{DM}$ & 10.744 & 63 & - \\
\hline II5 & $\mathrm{CA}$ & $\mathrm{F}$ & NA & 31.2 & DM & 5.069 & 42 & - \\
\hline II6 & CA & M & 55 & 32.8 & DM & 15.702 & 142 & - \\
\hline II7 & $\mathrm{CA}$ & $\mathrm{F}$ & NA & 28.4 & DM & 8.65 & 69 & OHA \\
\hline II8 & $\mathrm{CC}$ & M & - & 31.3 & NG & 5.124 & NA & - \\
\hline II9 & $\mathrm{CC}$ & $\mathrm{F}$ & 48 & 35.9 & DM & 6.942 & 81 & OHA \\
\hline II10 & $\mathrm{CA}$ & M & 52 & NA & DM & NA & NA & OHA, ins \\
\hline II11 & $\mathrm{CC}$ & M & 53 & 19.1 & PD & 5.014 & 39 & - \\
\hline III1 & CA & M & 33 & NA & DM & 6.612 & NA & - \\
\hline III2 & CA & M & - & 20.3 & $\mathrm{NG}$ & 4.408 & NA & - \\
\hline III3 & $\mathrm{CA}$ & $\mathrm{F}$ & 23 & 36.7 & DM & 13.113 & 98 & OHA \\
\hline III4 & CA & $\mathrm{F}$ & 29 & 36.3 & $\mathrm{DM}$ & 9.091 & 53 & - \\
\hline
\end{tabular}

B, Family B

\begin{tabular}{|c|c|c|c|c|c|c|c|c|}
\hline Member & Genotype & Sex & $\begin{array}{c}\text { Age at } \\
\text { diagnosis } \\
\text { (years) }\end{array}$ & $\begin{array}{c}\text { BMI } \\
\left(\mathrm{km} / \mathrm{m}^{2}\right)\end{array}$ & $\begin{array}{l}\text { Glycemic } \\
\text { status }\end{array}$ & $\begin{array}{c}\text { FPG } \\
(\mathrm{mmol} / \mathrm{l})\end{array}$ & $\begin{array}{c}\text { HbAlc } \\
(\mathrm{mmol} / \mathrm{mol})\end{array}$ & $\begin{array}{l}\text { Current } \\
\text { treatment }\end{array}$ \\
\hline I1 & $\mathrm{CA}$ & $\mathrm{M}$ & 40 & NA & $\mathrm{PD}$ & 5.675 & NA & - \\
\hline I2 & $\mathrm{CC}$ & $\mathrm{F}$ & - & NA & $\mathrm{NG}$ & 4.904 & NA & - \\
\hline II1 & $\mathrm{CA}$ & M & 18 & 27.3 & DM & 11.295 & NA & OHA \\
\hline $\mathrm{II} 2$ & $\mathrm{CC}$ & $\mathrm{F}$ & - & NA & NG & 4.628 & NA & - \\
\hline
\end{tabular}

Prediabetic status was indicated by $\mathrm{HbAlc} \geq 39 \mathrm{mmol} / \mathrm{mol}$, according to the ADA criteria. BMI, body mass index; DM, diabetes mellitus; NG, normal glucose; PD, prediabetic; FPG, fasting plasma glucose; HbA1c, glycated hemoglobin; ins, insulin; NA, not available; OHA, oral hypoglycemic agents.

Three-dimensional (3D) structure of protein and in silico mutagenesis. The 3D structure of the wild-type human DnaJ homolog subfamily C member 3 (DNAJC3) protein (2Y4T) (21) was obtained from The Protein Data Bank (http://www.rcsb. org/) (22). In silico mutagenesis and polar contact displays were performed using the PyMOL software version 1.8.4.0-r2 (Schrödinger, LLC, NY, USA).

Cell culture. The human pancreatic $\beta$-cell line $1.1 \mathrm{~b} 4$ was purchased from the American Type Culture Collection (Manassas, VA, USA). Cells were grown in RPMI-1640 medium (Invitrogen; Thermo Fisher Scientific, Inc., Waltham, MA, USA) containing 10\% fetal bovine serum (Gibco; Thermo Fisher Scientific, Inc.) and $1 \%$ antibiotics (100 IU/ml penicillin and $100 \mathrm{mg} / \mathrm{ml}$ streptomycin) at $37^{\circ} \mathrm{C}$ in a humidified atmosphere containing $5 \% \mathrm{CO}_{2}$.
Plasmid construct and transfection. In order to generate DNAJC3 plasmids, a DNAJC3 coding sequence (NM_006260) and a FLAG-tag sequence (DYKDDDDK) were amplified and cloned into the pcDNA3.1 plasmid (Invitrogen; Thermo Fisher Scientific, Inc.). All plasmids were amplified in E. coli (D5 $\alpha$ ) (Invitrogen; Thermo Fisher Scientific, Inc.) and purified using a mini kit (Qiagen GmbH, Hilden, Germany). For generating the DNAJC3 p.H238N clone, wild-type DNAJC3 plasmids were used as the template the wild-type was subjected to mutagenesis to obtain the mutant plasmid. On the day prior to transfection, $2 \times 10^{5}$ cells/well were seeded into 6 -well plates. After $24 \mathrm{~h}$, cells were transfected by $1 \mu \mathrm{g}$ DNAJC3 wild-type or p.H238N plasmid using Lipofectamine ${ }^{\circledR} 2000$ reagent (Invitrogen; Thermo Fisher Scientific, Inc.) according to manufacturer's protocol. After $48 \mathrm{~h}$ of transfection, cells were harvested and the stability of protein was measured by western 


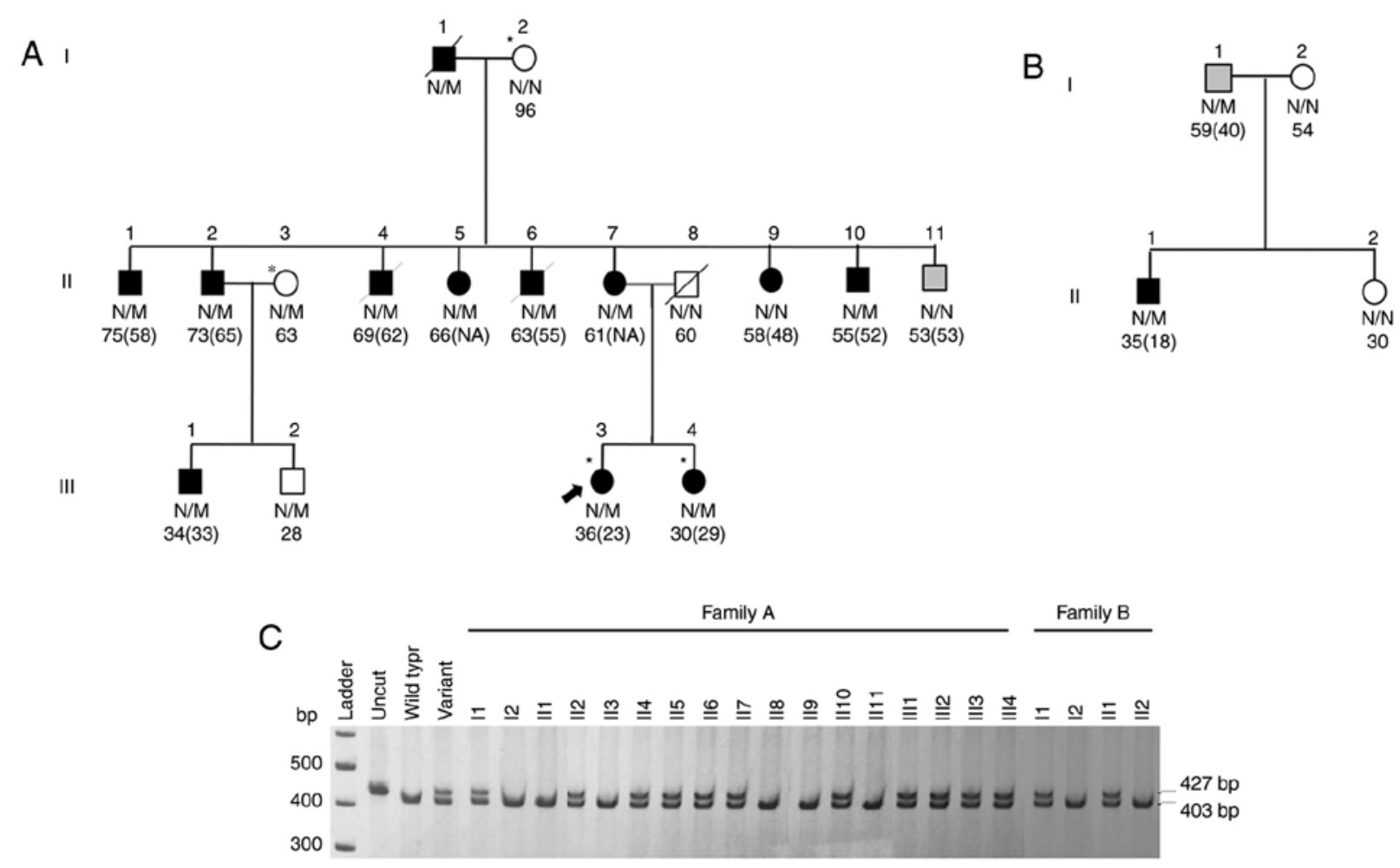

Figure 1. Segregation of DNAJC3 p.H238N with diabetes in type 2 diabetic families. The results are shown for (A) family A and (B) family B. Roman numerals on the upper left side indicate the generation numbers of the pedigree, and the numbers above each symbol indicate the individual ID within the generation. Males and females are indicated by squares and circles, respectively. Black-filled, gray-filled and open symbols represent the diabetic, prediabetic and non-diabetic subjects, respectively. The arrow indicates the proband (III3), while asterisks (*) indicate the samples subjected to exome sequencing. (C) Results of polymerase chain reaction-restriction fragment length polymorphism genotyping of DNAJC3 p.H238N in all family members. DNAJC3, DnaJ homolog subfamily C member 3; N/N, wild-type; N/M, variant.

blot analysis. The efficiency of plasmid transfection was determined using quantitative PCR by detection Neomycin DNA region of pcDNA3.1 plasmid. The forward primer (TGAATG AACTGCAGGACGAG) and reverse primer (ATACTTTCT CGGCAGGAGCA) were used to amplified the PCR product. qPCR was performed in a LightCycler 480 Instrument (Roche Diagnostics, Mannheim, Germany) using LightCycler ${ }^{\circledR} 480$ SYBR Green I Master (Roche Diagnostics, Mannheim, Germany). Initial enzyme activation proceeded at $95^{\circ} \mathrm{C}$ for $10 \mathrm{~min}$, followed by 45 cycles at $95^{\circ} \mathrm{C}$ for $30 \mathrm{sec}, 60^{\circ} \mathrm{C}$ for $20 \mathrm{sec}$, and $72^{\circ} \mathrm{C}$ for $20 \mathrm{sec}$. $\beta$-actin was used as an internal control to normalize input cDNA. Relative mRNA expression levels were calculated using the $2^{-\Delta \Delta \mathrm{Cq}}$ method (23). The assay was performed in triplicate.

RNA isolation and RNA transcripts level measurement. Total RNA from $1.1 \mathrm{~b} 4$ cells transfected with DNAJC3 wild-type, p.H238N, and empty pcDNA3.1 plasmid constructs were isolated using TRizol ${ }^{\circledR}$ reagent (Invitrogen; Thermo Fisher Scientific, Inc.). RNA was reverse-transcribed into cDNA using a Thermo Scientific Revert Aid First Strand cDNA Synthesis kit (Thermo Fisher Scientific, Inc.) and quantitated by means of qPCR (forward primer: 5'-GTCCTCTCTGAT CCAGAAATGA-3' and reverse primer 5'-TCATCGTCTTTG TAGTCATTGAAG-3'). The materials, methods, instrument, and calculation used for quantitative PCR were the same as those previously described for detection of the Neomycin DNA region.
Western blot analysis. Cells lysis was performed with a radioimmunoprecipitation assay reagent (Thermo Fisher Scientific, Inc.). Protein concentrations were then quantified using a NanoDrop 2000 spectrophotometer (Thermo Fisher Scientific, Inc., Wilmington, DE, USA) by Bradford protein assay (Bio-Rad Laboratories, Inc., Hercules, CA, USA). Proteins were separated on a $10 \%$ SDS-polyacrylamide gel by electrophoresis and then electroblotted to a nitrocellulose membrane (Bio-Rad Laboratories, Inc., Hercules, CA, USA) by a Semi-Dry Electrophoretic Transfer Cell system (Bio-Rad Laboratories, Inc., Hercules, CA, USA). The membrane was blocked with 5\% BSA (Sigma-Aldrich; Merck KGaA, Darmstadt, Germany) for $1 \mathrm{~h}$ at room temperature. Subsequently, the samples were incubated with primary mouse monoclonal antibody against FLAG (cat. no. F3165; 1:2,000; Sigma-Aldrich; Merck KGaA, Darmstadt, Germany) for $2 \mathrm{~h}$ and mouse monoclonal antibody against $\beta$-actin (cat. no. s-47778; 1:1,000; Santa Cruz Biotechnology, Inc., Dallas, TX, USA) for $1 \mathrm{~h}$ at room temperature. Membranes were incubated with horseradish peroxidase-linked goat anti-mouse secondary antibody (cat. no. P044701-2; 1:1,000; Dako; Agilent Technologies, Inc., Santa Clara, CA, USA). The binding antibodies were visualized using an enhanced chemiluminescence detection kit (SuperSignal ${ }^{\mathrm{TM}}$ West Pico PLUS Chemiluminescent Substrate; Thermo Fisher Scientific, Inc.) and bands were detected by Biomolecular Imager (ImageQuant LAS 4010; GE Healthcare Life Sciences, Little Chalfont, UK). $\beta$-actin staining was utilized as an internal control in all experiments. The expressed bands were 
quantified by computer-assisted scanning densitometry using ImageJ software version 1.4.3.x (National Institutes of Health, Bethesda, MD, USA). The experiments were conducted three times independently.

Statistical analysis. SPSS software version 17.0 (SPSS, Inc., Chicago, IL, USA) was used for data analysis. A $\chi^{2}$ test was used to examine the significance of the association between each variation and T2D. In case of a sample size of $<5$, Fisher's exact test was used to validate the association. A Student's t-test was conducted to evaluate mean differences in protein expression between the wild-type and mutant groups following transfection of the plasmids into $1.1 \mathrm{~b} 4 \beta$-cells. A P-value of $\leq 0.05$ was considered to indicate a statistically significant difference.

\section{Results}

Exome sequencing performance. An average of $2.8 \mathrm{~Gb}$ on-target yields were generated from each sample of four family members (data not shown), including two subjects with diabetes (III3 and III4) and two with normal glucose tolerance (I2 and II3). These four family members were selected for exome sequencing as representatives of this large early-onset T2D family because they were able to provide valuable information regarding diabetes-predisposing variants. The mean read depth of the target region was $45 x$, while 93 and $83 \%$ of the target region were covered more than 1 and 10x which mean that 93 and $83 \%$ of each base in the target region were at least 1 and 10x, respectively. A total of 72,723, 61,409, 64,723 and 76,413 sequence variants were detected in the samples from the I2, II3, III3 and III4 subjects, respectively. Among those, 3,451 variants were identified only in diabetic (III3 and III4) family members.

Candidate variant selection and validation. The number of candidate variants falling under each selection criteria is shown in Table II. In total, 152 of those variants were novel or rare heterozygous non-synonymous variants $(\mathrm{MAF}<0.01)$. Among these, 122 variants from 117 genes were predicted as having deleterious effects on protein functions by at least one of the four in silico mutation prediction programs. Furthermore, of these 122 variants, the GeneDistiller software revealed that 34 variants residing in 32 genes were implicated in diabetes and metabolism.

Segregation analysis. The 122 variants which were considered as possible pathogenic variants were subjected to segregation analysis with diabetes in family $\mathrm{A}$. This analysis indicated that a missense variant in DNAJC3 (NM_006260; c.C712A p.H238N) exhibited partial segregation with diabetes in this family (Fig. 1A and C). DNAJC3 encodes a co-chaperone of $\mathrm{BiP}$ and is involved in diabetes and metabolism, as demonstrated using the GeneDistiller software. Mutations of DNAJC3 have been attributed to DM, as well as multisystem neurodegeneration (24). DNAJC3 H238N was reported as rs527330902 in the Exome Aggregation Consortium (http://exac.broadinstitute.org/) database, with a frequency of $0.003 \%$ (3 out of a total of 119,086 alleles). Subsequent genotyping this variant in other 90 early-onset T2D/MODY $\mathrm{X}$ probands, demonstrated that DNAJC3 p.H238N segregated
Table II. Number of candidate variants under different selection criteria.

\begin{tabular}{lc}
\hline Selection criteria & $\begin{array}{c}\text { Number } \\
\text { of variants }\end{array}$ \\
\hline Variants from exome sequencing & 68,817 \\
Variants identified only in two & 3,451 \\
diabetic samples & 2,551 \\
Coding variants & 393 \\
Non-synonymous variants & 245 \\
Heterozygous non-synonymous variants & 152 \\
Heterozygous non-synonymous & 122 \\
variants with MAF <0.01 & \\
Variants predicted as having deleterious & \\
effect on protein function by in silico & \\
programs & DNAJC3 p.H238N \\
Segregation analysis & \\
\hline MAF, minor allele frequency. & \\
\hline
\end{tabular}

with diabetes in another family (Fig. 1B and C and Table I) but not in the other 89 early-onset T2D/MODYX probands.

Prevalence of DNAJC3 p.H238N in unrelated T2D patients and non-diabetic subjects. DNAJC3 p.H238N was also genotyped in unrelated T2D patients and individuals with normal glucose tolerance. This variant was identified in 14 out of $1,000 \mathrm{~T} 2 \mathrm{D}$ patients $(\mathrm{MAF}=0.007)$, in 2 out of 500 non-diabetic subjects $(\mathrm{MAF}=0.002)$, and in 3 prediabetic individuals who had previously been classified as non-diabetic subjects. In addition, DNAJC3 p.H238N exhibited a trend towards increasing the risk of developing T2D (odds ratio, 3.519; 95\% confidence interval, 0.80-15.62; Table III), although a statistically significant difference was not observed $(\mathrm{P}=0.107)$.

Functional impact of the mutation. Of the 4 in silico programs, used for prediction of functional impact of the mutation, 3 suggested DNAJC3 p.H238N as a deleterious mutation. These included the results from Mutation taster (score $=0.99$; cut-off $>0.5$ ), Variowatch (protein domain abolish), Polyphen2 (score=0.988; cut-off $>0.5$ ). In addition, DUET algorithms predicted instability of the mutant protein $(\Delta \Delta \mathrm{G}=-2.009 \mathrm{kcal} / \mathrm{mol}$; Table IV).

$3 D$ structure of mutant protein. DNAJC3 comprises 504 amino acids which are divided into an N-terminal ER-targeting tetratricopeptide repeat (TPR) domain, a peptide sequence that has 9 TPR motifs and C-terminal J domains (21). The crystal structure revealed the presence of 9 TPR motifs forming 3 TPR subdomains (21). Histidine residue at position 238 (H238) residing in TPR6 of subdomain II is conserved among humans, chimpanzees, elephants, cows, dogs, mice, rats, chicken, frogs and zebrafish (Fig. 2). Using the PyMol software, a polar contact between H238 and N269 of TPR7 of the protein subdomain III was detected. However, substitution of $\mathrm{H} 238$ with $238 \mathrm{~N}$ disturbed the polar contact by creating a 
Table III. Genotype and allele frequencies of DNAJC3 p.H238N identified in Thai subjects, including 1,000 type 2 diabetic patients and 500 non-diabetic subjects.

\begin{tabular}{|c|c|c|c|c|c|c|c|}
\hline \multirow[b]{2}{*}{ Genotypes } & \multicolumn{2}{|c|}{ Genotype frequencies, n (\%) } & \multirow[b]{2}{*}{ P-value } & \multicolumn{2}{|c|}{ Allele frequencies (\%) } & \multirow[b]{2}{*}{ Non-diabetic subjects } & \multirow[b]{2}{*}{ P-value } \\
\hline & $\mathrm{T} 2 \mathrm{D}$ & Non-diabetic subjects & & OR $(95 \% \mathrm{CI})$ & $\mathrm{T} 2 \mathrm{D}$ & & \\
\hline $\mathrm{CC}$ & 986 (98.6) & 498 (99.6) & 0.107 & $3.519(0.80-15.62)$ & C: 99.3 & C: 99.8 & 0.108 \\
\hline $\mathrm{CA}$ & $14(1.4)$ & $2(0.4)$ & & & A: 0.7 & A: 0.2 & \\
\hline AA & $0(0)$ & $0(0)$ & & & & & \\
\hline Total & $1,000(100)$ & $500(100)$ & & & & & \\
\hline
\end{tabular}

The location investigated was exon 6 , and the position (base change) was C712A.

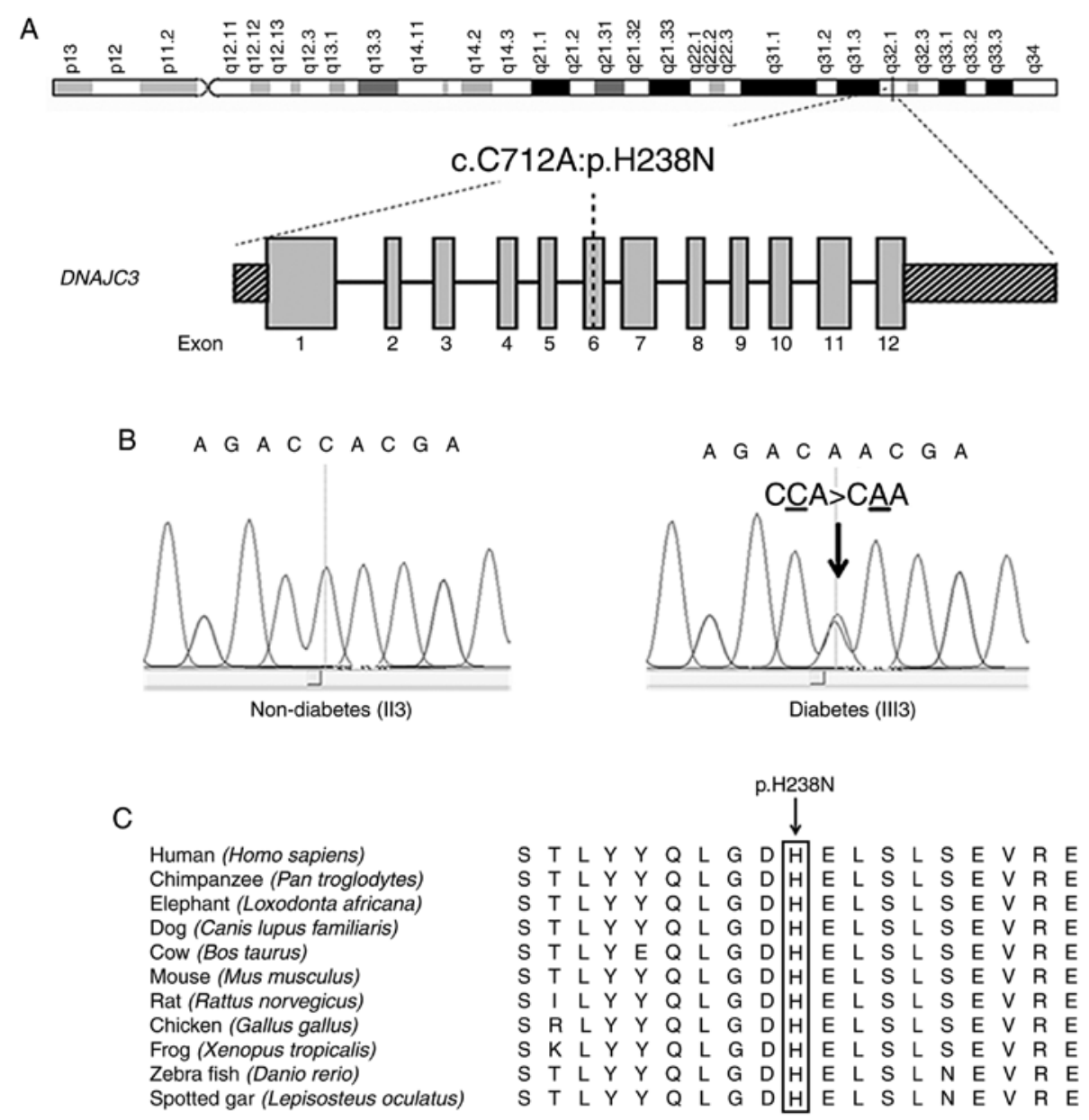

Figure 2. (A) Position of DNAJC3 p.H238N on chromosome 13. (B) Sanger sequencing of DNAJC3 p.H238N obtained from diabetic (III3) and non-diabetic (II3) family members. The evolutionary conservation of DNAJC3 p.H238N (C) is shown. DNAJC3, DnaJ homolog subfamily C member 3.

new, stronger hydrogen bond with Y304, residing in the region between TPR7 and TPR8 of subdomain III (Fig. 3).

Protein stability of DNAJC3 p.H238N. DNAJC3 wild-type and mutant plasmid tag with FLAG sequence were constructed for detection of the expression of DNAJC3 p.H238N protein without the effect of endogenous protein in the $1.1 \mathrm{~b} 4$ human pancreatic $\beta$-cell line. The results demonstrated that the expression of DNAJC3 p.H238N protein was significantly lower compared with that of the wild-type $(0.68 \pm 0.08$-fold change; $\mathrm{P}<0.05$; Fig. 4). Furthermore, the efficiency of plasmid transfection was not significantly different between DNAJC3 wild-type and mutant plasmid (data not shown).

\section{Discussion}

The aim of the current study was to identify a pathogenic mutation causing diabetes in a large Thai multigenerational pedigree with diabetic family members in 3 generations, consistent with an autosomal dominant mode of inheritance by 
Table IV. Bioinformatics analysis of DNAJC3 p.H238N.

\begin{tabular}{llcc}
\hline Program & \multicolumn{1}{c}{ Prediction } & Score & Cut-off value \\
\hline $\begin{array}{l}\text { MutationTaster } \\
\text { VarioWatch }\end{array}$ & $\begin{array}{l}\text { Disease causing } \\
\text { High, missense } \\
\text { (protein domain abolished) }\end{array}$ & 0.99 & $>0.5$ \\
PolyPhen2 & Deleterious & - & $>0.5$ \\
SIFT & Tolerated & 0.31 & $<0.05$ \\
DUET (predicted stability changes) & Destabilizing & $\Delta \Delta \mathrm{G}=-2.009 \mathrm{kcal} / \mathrm{mol}$ & $\begin{array}{l}\text { Negative values denote } \\
\text { destabilizing mutations }\end{array}$
\end{tabular}

Mutation was predicted using online prediction tools. DUET is a web server that uses an integrated computational approach to study missense mutations in proteins (http://structure.bioc.cam.ac.uk/duet).

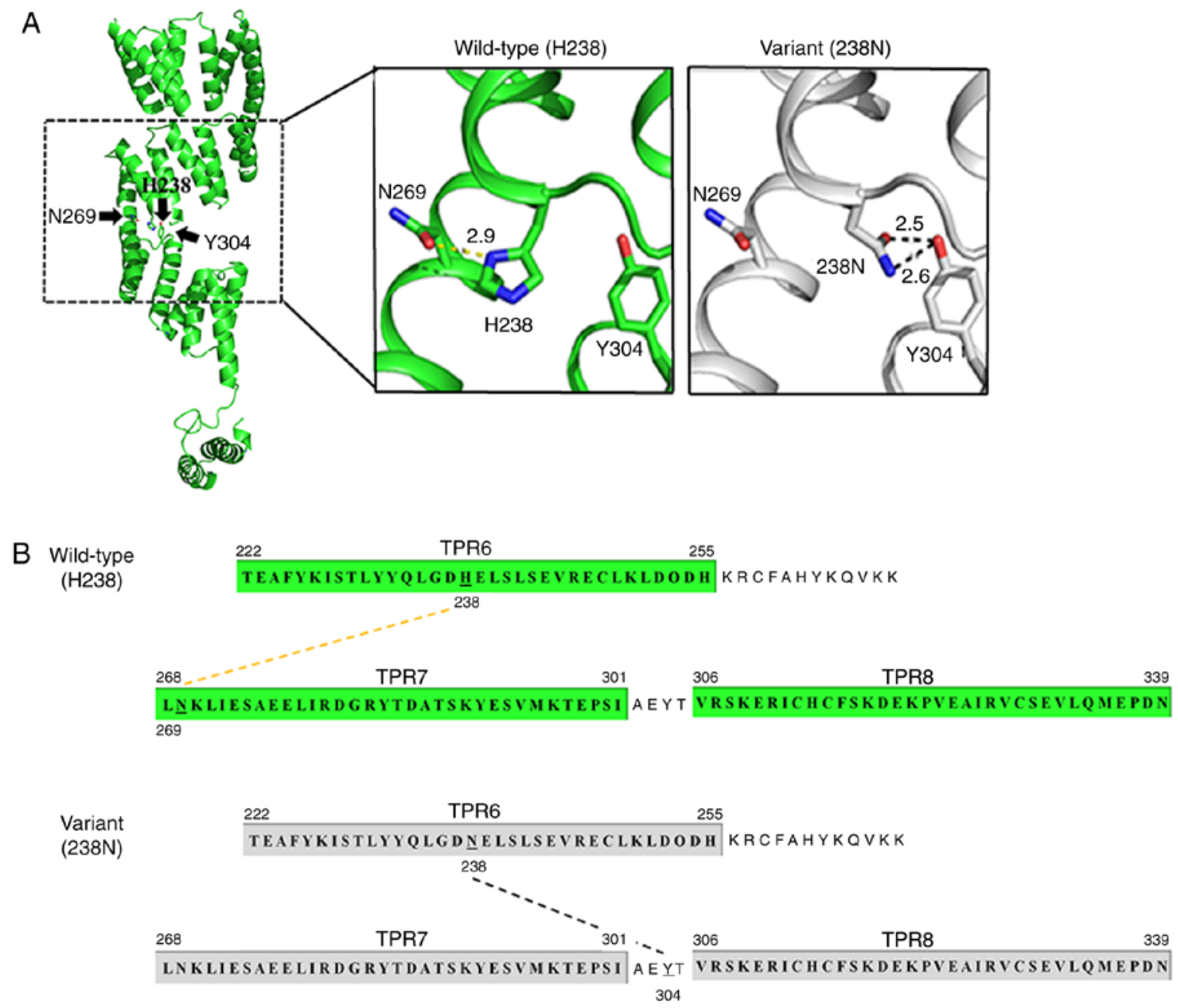

Figure 3. Local structural alteration due to amino acid substitution H238N of DNAJC3. (A) Three-dimensional structure of DNAJC3. DNAJC3 wild-type and variant $\mathrm{H} 238 \mathrm{~N}$ are shown in green and grey, respectively. Dotted lines represent the polar contacts between residues. Oxygen and nitrogen atoms are presented in red and blue, respectively. Numbers indicate the distance between atoms in Armstrong ( $\AA$ ). (B) Amino acid residues contributed to TPR6, TPR7 and TPR8 of DNAJC3. Dotted lines represent polar contacts between DNAJC3 H238N and neighbor residues. DNAJC3, DnaJ homolog subfamily C member 3; TPR, tetratricopeptide repeat.

exome sequencing. The proband (female), the proband's sibling (female) and one of their relatives in generation III were diagnosed with diabetes before 35 years of age. The identified rare DNAJC3 p.H238N was partially segregated with diabetes in the family. Although subject III2 carried DNAJC3 p.H238N, he exhibited a normal fasting plasma glucose level. The patient was young (28-years-old) and had a normal BMI at the time of diagnosis; therefore, this patient may develop diabetes later in life, or due to being overweight or obese. The other two diabetic family members (II1 and II9) who did not carry the 


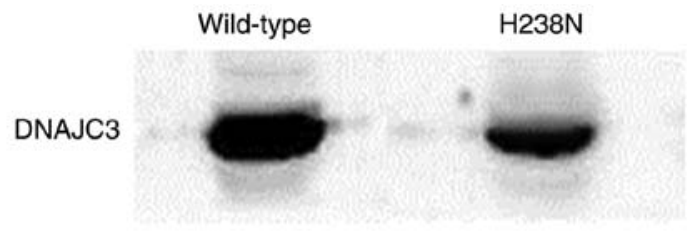

$\beta$-actin
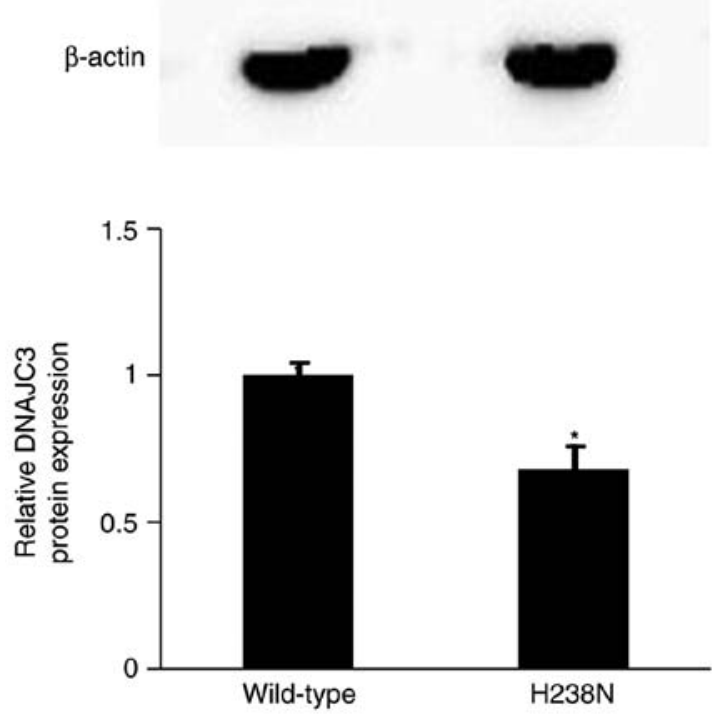

Figure 4. Protein expression of DNAJC3 wild-type and mutant plasmids detected by western blot analysis. "P $<0.05$ vs. wild-type. DNAJC3, DnaJ homolog subfamily $\mathrm{C}$ member 3 .

DNAJC3 mutation were probably phenocopies $(25,26)$. In subject II11 (male), although DNAJC3 p.H238N was detected, the patient displayed a normal fasting plasma glucose level ( $4.959 \mathrm{mmol} / \mathrm{l}$ ); however, the HbA1c level of this patient was in the prediabetes range $(39 \mathrm{mmol} / \mathrm{mol})$. Therefore, follow-up of the glucose tolerance status is mandatory. In addition, this variant was identified in another early-onset T2D family without mutation of known MODY genes in which diabetes had been transmitted from father to son, emphasizing the important role of DNAJC3 p.H238N and glycemic traits. Furthermore, DNAJC3 p.H238N was identified in unrelated T2D patients $(\mathrm{MAF}=0.007)$ and in non-diabetic subjects $(\mathrm{MAF}=0.002)$, as well as 3 prediabetes cases. However, the allele frequencies of DNAJC3 p.H238N in T2D and non-diabetic subjects were not statistically different, which is possibly due to the relatively small sample size. It is possible that there are modifier genes or environmental factors that can alter the effect or penetrance of this variant. The results of the current study implied that this variant was neither a rare allele causing a Mendelian disease, nor a common variant implicated in the common T2D. Instead, it is a low-frequency variant with an intermediate effect that confers a higher risk of developing a chronic disease (such as T2D), as described by Manolio et al (27).

$D N A J C 3$ mRNA and protein are expressed in the pancreatic $\beta$-cells. This protein is involved in an unfolded protein response (UPR) during endoplasmic reticulum (ER) stress (28). The ER can transmit apoptotic signals in the pancreas during ER stress, which mediates the apoptosis of $\beta$-cells during the development of diabetes (29-32). Under the ER stress condition, cells activate the adaptive UPR to resolve the protein-folding defect (33). In addition, an increased expression of UPR genes is observed in the islets of T2D patients $(34,35)$. Several studies have indicated that DNAJC3 is regularly induced during ER stress, which is a key component of a negative feedback used by the cell to inhibit eukaryotic initiation factor-2 signaling and attenuate the UPR, leading to a reduction of cell apoptosis $(36,37)$. Ladiges et al $(28)$ generated DnajC3 knockout mice, demonstrating an ongoing onset of glycosuria and hyperglycemia associated with an increasing apoptosis of the pancreatic islet cells. A recent study by Han et al (38) revealed that defective protein folding generated oxidative stress, which serves as an essential signal for apoptosis in response to ER stress in the pancreatic $\beta$-cells. The authors demonstrated that homozygous DNAJC3 knockout mice become diabetic due to decreased $\beta$-cell mass and function as a result of the oxidative stress and apoptosis signaling (38). Furthermore, Synofzik et al (24) demonstrated that a homozygous DNAJC3 p.R194* mutation identified in three siblings from a Turkish family caused juvenile-onset diabetes with central and peripheral neurodegeneration. Also, they discovered that the homozygous frameshift deletion DNAJC3 p.N34Mfs*20 caused juvenile-onset diabetes with hearing impairment and ataxia in a German family with a consanguineous marriage (24). These two diabetes-associated mutations caused loss of the DNAJC3 protein function. Furthermore, 39 missense homozygous variants of DNAJC 3 were identified in 506 unrelated subjects with a family history consistent with recessive disease, with one or more features of the DNAJC3 phenotype in this cluster (diabetes, neurodegeneration, hearing impairment or ataxia) (24).

Notably, the present study identified DNAJC3 p.H238N, which segregated with diabetes in autosomal dominant mode. Since it was a heterozygous mutation, the disruption of the protein function may be less harmful compared with its homozygous counterpart. In silico mutagenesis of DNAJC $3 \mathrm{H} 238 \mathrm{~N}$ revealed alteration of the polar contact across TPR motifs and subdomains, which may affect the tertiary structure of the DNAJC3 protein. The DNAJC3 RNA levels of cells transfected with the wild-type and mutant plasmid constructs were not statistically different (data not shown). By contrast, the expression of DNAJC3 $\mathrm{H} 238 \mathrm{~N}$ protein was lower compared with that in the wild-type, which may be due to protein instability. This is consistent with the 'Destabilizing' result predicted by DUET predicted stability changes program. Since DNAJC3 R194* and N34Mfs*20 caused loss of protein expression and function, the clinical manifestations of patients harboring these mutations were more severe in comparison with those harboring DNAJC3 p.H238N (24). Thus, the patients in the present study became diabetic at a later age and did not exhibit neurodegeneration, hearing impairment or ataxia. Further studies considering the molecular and cell biology of this variant, particularly in pancreatic $\beta$-cells, should promote a better understanding of the mechanism underlying the pathogenesis of T2D, and such research may lead to the development of novel therapies for DM. Furthermore, screening for this variant in a larger sample size of T2D patients and non-diabetic subjects should be conducted to clarify the association between this variant and the risk of developing diabetes.

In conclusion, in the present study, exome sequencing successfully identified DNAJC3 p.H238N, which is a 
low-frequency variant with an intermediate effect, causing familial T2D in Thai individuals. The findings of the present study were the first to demonstrate that DNAJC3 variants were involved in pathogenesis of early-onset autosomal dominant T2D while a previous report identified that different variants of this gene were involved in syndromic autosomal recessive diseases with diabetes (24).

\section{Acknowledgements}

Not applicable.

\section{Funding}

This research project was supported by the Mahidol University Grant, Siriraj Research Grant for Research and Development, Faculty of Medicine, Siriraj Hospital (awarded to NP, WT, PJ and CC), the Thailand Research Fund (grant nos. BRG5280008, TRG5780113 and IRG5980006; awarded to NP, WT and PTY), and a Mahidol University Postdoctoral Fellowship Grant (awarded to SK). PJ and PY were supported by a Chalermprakiat Grant from the Faculty of Medicine, Siriraj Hospital.

\section{Availability of data and materials}

The datasets used and/or analyzed during the current study are available from the corresponding author on reasonable request.

\section{Authors' contributions}

SK, PTY and NP conceived and designed the study. WT and PJ analyzed and interpreted the patient data and modeling. CC, NB and JS performed the cell experiments and molecular biology analysis. SK was a major contributor in writing the manuscript. All authors read and approved the final manuscript.

\section{Ethics approval and consent to participate}

The present study was approved by the Ethics Committee, Faculty of Medicine, Siriraj Hospital, Mahidol University (COA no. Si491/2014). Written informed consent was obtained.

\section{Consent for publication}

All subjects were informed of the purpose of the study prior to signing an informed consent form.

\section{Competing interests}

The authors declare that they have no competing interests.

\section{References}

1. Guariguata L, Whiting DR, Hambleton I, Beagley J, Linnenkamp U and Shaw JE: Global estimates of diabetes prevalence for 2013 and projections for 2035. Diabetes Res Clin Pract 103: 137-149, 2014.
2. Roglic G, Unwin N, Bennett PH, Mathers C, Tuomilehto J, Nag S, Connolly $\mathrm{V}$ and King $\mathrm{H}$ : The burden of mortality attributable to diabetes: Realistic estimates for the year 2000. Diabetes Care 28: 2130-2135, 2005

3. Murea M, Ma L and Freedman BI: Genetic and environmental factors associated with type 2 diabetes and diabetic vascular complications. Rev Diabet Stud 9: 6-22, 2012.

4. Wilmot $\mathrm{E}$ and Idris I: Early onset type 2 diabetes: Risk factors, clinical impact and management. Ther Adv Chronic Dis 5: 234-244, 2014.

5. Bridger T: Childhood obesity and cardiovascular disease. Paediatr Child Health 14: 177-182, 2009.

6. Fajans SS, Bell GI and Polonsky KS: Molecular mechanisms and clinical pathophysiology of maturity-onset diabetes of the young. N Engl J Med 345: 971-980, 2001.

7. Sanghera DK and Blackett PR: Type 2 diabetes genetics: Beyond GWAS. J Diabetes Metab 3: 6948, 2012.

8. Lee S, Abecasis GR, Boehnke M and Lin X: Rare-variant association analysis: Study designs and statistical tests. Am J Hum Genet 95: 5-23, 2014.

9. Morris AP, Voight BF, Teslovich TM, Ferreira T, Segrè AV, Steinthorsdottir V, Strawbridge RJ, Khan H, Grallert H, Mahajan A, et al: Large-scale association analysis provides insights into the genetic architecture and pathophysiology of type 2 diabetes. Nat Genet 44: 981-990, 2012.

10. Cirulli ET and Goldstein DB: Uncovering the roles of rare variants in common disease through whole-genome sequencing. Nat Rev Genet 11: 415-425, 2010.

11. Lohmueller KE, Spars $\varnothing$ T, Li Q, Andersson E, Korneliussen T, Albrechtsen A, Banasik K, Grarup N, Hallgrimsdottir I, Kiil K, et al: Whole-exome sequencing of 2,000 Danish individuals and the role of rare coding variants in type 2 diabetes. Am J Hum Genet 93: 1072-1086, 2013.

12. Kwak SH, Jung CH, Ahn CH, Park J, Chae J, Jung HS, Cho YM, Lee DH, Kim JI and Park KS: Clinical whole exome sequencing in early onset diabetes patients. Diabetes Res Clin Pract 122: 71-77, 2016.

13. Plengvidhya $\mathrm{N}$, Boonyasrisawat $\mathrm{W}$, Chongjaroen $\mathrm{N}$, Jungtrakoon P, Sriussadaporn S, Vannaseang S, Banchuin N and Yenchitsomanus PT: Mutations of maturity-onset diabetes of the young (MODY) genes in Thais with early-onset type 2 diabetes mellitus. Clin Endocrinol (Oxf) 70: 847-853, 2009.

14. Standards of medical care in diabetes-2017: Summary of revisions. Diabetes care 40: S4-S5, 2017.

15. Li H and Durbin R: Fast and accurate short read alignment with Burrows-Wheeler transform. Bioinformatics 25: 1754-1760, 2009.

16. Li H, Handsaker B, Wysoker A, Fennell T, Ruan J, Homer N, Marth G, Abecasis G and Durbin R; 1000 Genome Project Data Processing Subgroup: The sequence alignment/map format and SAMtools. Bioinformatics 25: 2078-2079, 2009.

17. McKenna A, Hanna M, Banks E, Sivachenko A, Cibulskis K, Kernytsky A, Garimella K, Altshuler D, Gabriel S, Daly M and DePristo MA: The genome analysis toolkit: A mapreduce framework for analyzing next-generation DNA sequencing data. Genome Res 20: 1297-1303, 2010.

18. Dong C, Wei P, Jian X, Gibbs R, Boerwinkle E, Wang K and Liu X: Comparison and integration of deleteriousness prediction methods for nonsynonymous SNVs in whole exome sequencing studies. Hum Mol Genet 24: 2125-2137, 2015.

19. Seelow D, Schwarz JM and Schuelke M: GeneDistiller-distilling candidate genes from linkage intervals. PloS One 3: e3874, 2008.

20. Pires DE, Ascher DB and Blundell TL: DUET: A server for predicting effects of mutations on protein stability using an integrated computational approach. Nucleic Acids Res 42: W314-W319, 2014.

21. Svärd M, Biterova EI, Bourhis JM and Guy JE: Crystal structure of the human co-chaperone P58(IPK). PLoS One 6: e22337, 2011.

22. Berman HM, Westbrook J, Feng Z, Gilliland G, Bhat TN, Weissig H, Shindyalov IN and Bourne PE: The protein data bank. Nucleic Acids Res 28: 235-242, 2000.

23. Livak KJ and Schmittgen TD: Analysis of relative gene expression data using real-time quantitative PCR and the 2(-delta delta C(T)) method. Methods 25: 402-408, 2001.

24. Synofzik M, Haack TB, Kopajtich R, Gorza M, Rapaport D, Greiner M, Schönfeld C, Freiberg C, Schorr S, Holl RW, et al: Absence of BiP co-chaperone DNAJC3 causes diabetes mellitus and multisystemic neurodegeneration. Am J Hum Genet 95: 689-697, 2014. 
25. Yamagata K, Oda N, Kaisaki PJ,Menzel S, Furuta H, Vaxillaire M, Southam L, Cox RD, Lathrop GM, Boriraj VV, et al: Mutations in the hepatocyte nuclear factor-1alpha gene in maturity-onset diabetes of the young (MODY3). Nature 384: 455-458, 1996.

26. Stoffers DA, Ferrer J, Clarke WL and Habener JF: Early-onset type-II diabetes mellitus (MODY4) linked to IPF1. Nat Genet 17: 138-139, 1997.

27. Manolio TA, Collins FS, Cox NJ, Goldstein DB, Hindorff LA, Hunter DJ, McCarthy MI, Ramos EM, Cardon LR, Chakravarti A, et al: Finding the missing heritability of complex diseases. Nature 461: 747-753, 2009.

28. Ladiges WC, Knoblaugh SE, Morton JF, Korth MJ, Sopher BL, Baskin CR, MacAuley A, Goodman AG, LeBoeuf RC and Katze MG: Pancreatic $\beta$-cell failure and diabetes in mice with a deletion mutation of the endoplasmic reticulum molecular chaperone gene P58IPK. Diabetes 54: 1074-1081, 2005.

29. Mao C, Dong D, Little E, Luo S and Lee AS: Transgenic mouse model for monitoring endoplasmic reticulum stress in vivo. Nat Med 10: 1013-1014, 2004.

30. Iwawaki T, Akai R, Kohno K and Miura M: A transgenic mouse model for monitoring endoplasmic reticulum stress. Nat Med 10 98-102, 2004.

31. Ron D: Translational control in the endoplasmic reticulum stress response. J Clin Invest 110: 1383-1388, 2002.

32. Zhang $K$ and Kaufman RJ: Signaling the unfolded protein response from the endoplasmic reticulum. J Biol Chem 279: 25935-25938, 2004.
33. Manié SN, Lebeau J and Chevet E: Cellular mechanisms of endoplasmic reticulum stress signaling in health and disease. 3. Orchestrating the unfolded protein response in oncogenesis: An update. Am J Physiol Cell Physiol 307: C901-C907, 2014.

34. Huang CJ, Lin CY, Haataja L, Gurlo T, Butler AE, Rizza RA and Butler PC: High expression rates of human islet amyloid polypeptide induce endoplasmic reticulum stress mediated $\beta$-cell apoptosis, a characteristic of humans with type 2 but not type 1 diabetes. Diabetes 56: 2016-2027, 2007.

35. Laybutt DR, Preston AM, Akerfeldt MC, Kench JG, Busch AK, Biankin AV and Biden TJ: Endoplasmic reticulum stress contributes to $\beta$-cell apoptosis in type 2 diabetes. Diabetologia 50: 752-763, 2007.

36. van Huizen R, Martindale JL, Gorospe M and Holbrook NJ: P58IPK, a novel endoplasmic reticulum stress-inducible protein and potential negative regulator of eIF2alpha signaling. J Biol Chem 278: 15558-15564, 2003.

37. Yan W, Frank CL, Korth MJ, Sopher BL, Novoa I, Ron D and Katze MG: Control of PERK eIF2alpha kinase activity by the endoplasmic reticulum stress-induced molecular chaperone P58IPK. Proc Natl Acad Sci USA 99: 15920-15925, 2002.

38. Han J, Song B, Kim J, Kodali VK, Pottekat A, Wang M, Hassler J, Wang S, Pennathur S, Back SH, et al: Antioxidants complement the requirement for protein chaperone function to maintain $\beta$-cell function and glucose homeostasis. Diabetes 64: 2892-2904, 2015. 